\title{
Potential benefits of using ecological momentary assessment to study high-risk polydrug use
}

\author{
Alexis M. Roth ${ }^{1 *}$, Marisa Felsher ${ }^{1 *}$, Megan Reed ${ }^{1}$, Jesse L. Goldshear ${ }^{2}$, Quan Truong ${ }^{3}$, Richard S. \\ Garfein $^{4}$, Janie Simmons ${ }^{5}$ \\ ${ }^{1}$ Drexel University, Dornsife School of Public Health, Philadelphia, USA; ${ }^{2}$ Penn Medicine Center for Digital Health, University of Pennsylvania, \\ Philadelphia, USA; ${ }^{3}$ Palo Alto Medical Foundation Research Institute, Mountain View, CA, USA; ${ }^{4}$ Department of Medicine, Division of Global \\ Public Health, University of California, San Diego, USA; ${ }^{5}$ National Development and Research Institutes, Inc., New York, USA \\ Contributions: (I) Conception and design: AM Roth, J Simmons, R Garfein; (II) Administrative support: AM Roth; (III) Provision of study materials \\ or patients: AM Roth; (IV) Collection and assembly of data: AM Roth, JL Goldshear, Q Truong; (V) Data analysis and interpretation: AM Roth, M \\ Felsher, M Reed, JL Goldshear, Q Truong; (VI) Manuscript writing: All authors; (VII) Final approval of manuscript: All authors. \\ *These authors contributed equally to this work. \\ Correspondence to: Alexis M. Roth, PhD, MPH. Drexel University Dornsife School of Public Health, 3215 Market Street, Room 454, Philadelphia, PA \\ 19104, USA. Email: amr395@drexel.edu.
}

Background: While studies have documented both the feasibility and acceptability of using ecological momentary assessment (EMA) to study drug use, there is little empirical research assessing participants' perceptions of utilizing this technology-driven approach.

Methods: Participants were English-speaking persons $\geq 18$ years old who reported injection drug use and sequential (e.g., alcohol followed by opioid use) or simultaneous (i.e., injecting heroin and cocaine in one shot) polydrug use within 30 days recruited in San Diego, CA and Philadelphia, PA. Participants (N=36) completed two cell phone-based EMA simulations assessing mood, drug use, HIV risk behaviors, and daily activities, followed by semi-structured interviews that probed for potential benefits of participation over time. Qualitative analysis involved an iterative process of reviewing texts from the interviews to create a coding framework, which was then applied to all transcripts to identify themes.

Results: Findings suggest participants may derive indirect benefits from participation in EMA studies including: improved self-worth from helping others; experiencing increased social support through utilization of the study-provided mobile device for non-research purposes; and most importantly, increased self-reflection, which could lead to therapeutic and intervention-like effects such as decreased substance use or reduced HIV risk.

Conclusions: Participants identified a variety of potential benefits from participating in a study that utilizes EMA. This research suggests that benefits are highly salient for individuals involved in studies of polydrug use.

Keywords: Smartphone; injection drugs; ecological momentary assessment; ethicshealth behavior

Received: 08 July 2017; Accepted: 30 August 2017; Published: 30 October 2017.

doi: 10.21037/mhealth.2017.10.01

View this article at: http://dx.doi.org/10.21037/mhealth.2017.10.01

\section{Introduction}

Ecological momentary assessment (EMA) emerged as a critical research method in substance abuse and mood research beginning in the mid-1990s (1-4). EMA involves repeatedly assessing an individual's behaviors and experiences in real-time, as they go about their daily lives in their natural environment often on mobile phones or other cellular devices (5). EMA is particularly suitable for studying substance use, because it is episodic and thought to be 
related to mood and context (1). As a result of the specificity and reliability of data collected via EMA, there has been a dramatic increase in the use of EMA as a research tool with many studies focusing on vulnerable or marginalized populations reporting illegal and/or stigmatized behavior (6-10).

While studies have documented both the feasibility (11-14) and acceptability (6) of using EMA to study drug use, discussions regarding the ethics of using technologydriven data collection modalities common to EMA are just beginning to emerge. Thus far, concerns have been raised about the potential harms of capturing data about illicit and stigmatized behaviors of vulnerable populations (15) especially since EMA often includes geolocation of the risk activity of interest, and the provision of valuable mobile devices to collect EMA data may expose participants to physical targeting for theft (16). Thus, risks to privacy and confidentiality are highly salient. While repetitive questioning about mood or drug use might cause psychological issues (i.e., anxiety) (17) research participants have indicated that these risks (e.g., a trigger for drug relapse, physical harm stemming from theft of EMA phones, and social risks from being perceived by peers as a police informant) are unlikely but possible $(18,19)$.

Despite the growing body of literature on potential harms to study participants, the benefits that participants may receive from participation in EMA research have been less frequently assessed. Some researchers pose that "assessment reactivity," or shifts in behavior over time as it is intensely monitored during EMA studies, may be a benefit of this methodology (5). However, the studies that have explored assessment reactivity within the field of EMA have produced mixed results (20-23). For example, in the field of alcohol abuse research, Epstein et al. showed that assessment of alcohol use, mental health and affect via EMA triggers reduction in drinking pre-treatment among alcohol-dependent women (20), but Hufford et al.'s study of alcohol-abusing college students did not show changes in drinking frequency during EMA recording, relative to preEMA levels (22). Thus, additional research using designs to capture shifts in participant behavior over sustained periods of time are still needed to tease apart these effects.

The aim of the present study was to elicit participant perspectives on how participation in an EMA study of drug use might be beneficial to persons engaging in highrisk polydrug use. Our main research question was: what are the perceived benefits associated with participation in a hypothetical month-long EMA study of drug use?
Subsequent questions explored the processes by which these benefits could be conferred. Subsequent studies will employ a pre-post design with quantitative and qualitative assessments to test whether benefits manifest as participants hypothesize.

\section{Methods}

Data were collected from 36 participants in two United States cities (San Diego, CA and Philadelphia, PA) between May 2014 and April 2015. Eligibility criteria were: 18 years of age or older, injected illicit drugs at least once in the prior month, reported weekly sequential (i.e., alcohol followed by opioid use) or simultaneous (i.e., speedball or injecting heroin and cocaine in one shot) polydrug use and were able to provide informed consent. Subjects from the San Diego sample $(n=18)$ were recruited from STAHRII, a prospective, mixed-methods observational cohort study between May 2012 and July 2014 (24). The names of participants who reported polydrug use within 6 months during a STAHR-II study visit were randomly selected to be called for recruitment into the San Diego sample. Recruitment in Philadelphia ( $\mathrm{n}=18)$ occurred at Prevention Point Philadelphia (PPP), a not-for-profit agency that provides harm reduction services, including syringe exchange and overdose prevention services. Recruitment at PPP took place between February and April 2015. Flyers advertising the study were hung in public spaces. Participants who self-identified as polydrug users called the research team in order to be screened for eligibility. At both sites, participant socio-demographics and drug use were measured using technology-assisted surveys. An inventory of study instruments has been described in detail elsewhere (18).

During the course of the 90-minute study visit, participants completed two smartphone-based EMA simulations. Each EMA simulation lasted approximately 15 minutes and assessed recent mood, drug use, HIV risk behaviors, antiretroviral medication adherence (for persons living with HIV only), and daily activities (i.e., spending time with friends or family, providing childcare, doing household chores, etc.) (18). Simulations were followed by a semi-structured interview in which participants were asked to "think aloud" about their experience and reflections on potential benefits associated with completing the EMA twice daily for a span of 30 days $(25,26)$. Sample questions included, "Describe some of the good things that could happen to someone who participated in a daily diary study 
of drug use," and "Describe for me some concerns people might have about being given a cell phone to carry with them daily". Focusing on both potential benefits and potential harms associated with participation in EMA research allowed for a more comprehensive understanding of participant experiences. However, data on potential harms are reported elsewhere (18). All interviews were digitally recorded and transcribed verbatim. Participants were compensated $\$ 30$ upon completion. All participants gave informed consent. This study protocol was approved by the Drexel University and University of California San Diego Institutional Review Boards (protocol numbers 3192 and 3113 at Drexel University and UCSD protocol number 3192) as well as the Prevention Point Philadelphia Board of Directors, and it conforms to the provisions in accordance with the Helsinki Declaration.

Qualitative analysis involved an iterative process of reviewing texts from the interviews to create a coding framework. First, a priori or "structured codes" corresponding to the domains in the interview guides were developed. Second, 3-5 transcripts were read by three separate team members to develop a framework of "emergent codes" reflecting unanticipated themes from the interviews. Third, two research assistants independently applied the coding framework to the qualitative texts; a third researcher reviewed the coding framework for consistency until inter-rater reliability exceeded $75 \%$. In the final phase of analysis, exemplar quotes were selected for each qualitative theme and subtheme.

Quantitative analysis of survey data in both cities assessed: socio-demographic characteristics, drug and alcohol use, syringe and injection equipment sharing, and cell phone use. In San Diego, survey data was obtained from the participants' most recent STAHR-II quarterly follow-up visit. The survey data recall period was 6 months. In Philadelphia, these data were collected using a selfadministered electronic survey and the recall period was 3 months. All responses of "refuse to answer" were recoded as missing for purposes of calculating percentages. Statistical analyses were performed using SPSS, which included frequencies to describe the sample as well as chi square and $t$-tests to assess for differences between respondents by city (IBM Corp, 2013).

\section{Results}

Participants ( $\mathrm{n}=36)$ were primarily male $(63.9 \%)$, about half identified as White (55.6\%), with a median age of 45 .
The majority of participants $(77.8 \%)$ earned an annual income of less than $\$ 10,000$, and $55.6 \%$ were homeless. Two thirds of participants owned a cell phone at the time of the study, and of those who owned a cell phone, about half (54.2\%) owned a smartphone. Despite being recruited from opposite coasts of the U.S., participant socio-demographic characteristics were relatively similar across sites. Median age, gender, income, age at first injection, syringe sharing, and current cell phone ownership were not significantly different between participants in the two cities. Participants in San Diego reported higher educational attainment (72.2\% vs. $22.2 \%$ had some college education; $\mathrm{P}<0.01)$, reported more frequent binge drinking $(76.9 \%$ vs. $16.7 \%$ daily binge drinking; $\mathrm{P}<0.01$ ), and were more likely to report owning a smart phone $(78.6 \%$ compared to $11.1 \% ; \mathrm{P}<0.001)$. Drugs injected most often also varied by city $(\mathrm{P}<0.01)$. In San Diego, the most frequently injected drug was methamphetamine $(73.3 \%)$ while in Philadelphia the most frequently injected drug was heroin (82.4\%). Additional comparisons are shown in Table 1.

The subjects described a variety of potential benefits but most were psychological in nature, including increased self-reflection (which in turn could become a cue to action to both modify harmful behavior and reinforce healthy behaviors) altruism, catharsis, and increased social support via the study-provided cell phone. We describe each form of potential benefits in detail below.

\section{Self-reflection}

The majority of participants reported that taking part in an EMA study about drug use might cause them to reflect on their emotions, drug use and daily activities. For some, reporting their mood and behavior on a daily basis was considered a benefit. A 50-year-old male participant described, "If I'm doing this study, it would make me take a look at my own actions in a lot of areas, and I think that might be a good thing. A reality-check... it would be a good thing." Further, participants indicated completing daily assessments via a mobile device would be more beneficial than recounting daily activities and mood to a person. A 40-year-old male summarized:

"Doing the study on a cell phone (as opposed to one on one with an interview) is even better...because I feel like there's nobody that can judge me. I'm just answering it on the phone. I think that diary shit is pretty cool. If you did start like, where you would have to answer shit through the course of the day, it would keep you more aware of what you were doing." 
Table 1 Demographic and drug use factors by study site ${ }^{* \dagger}$

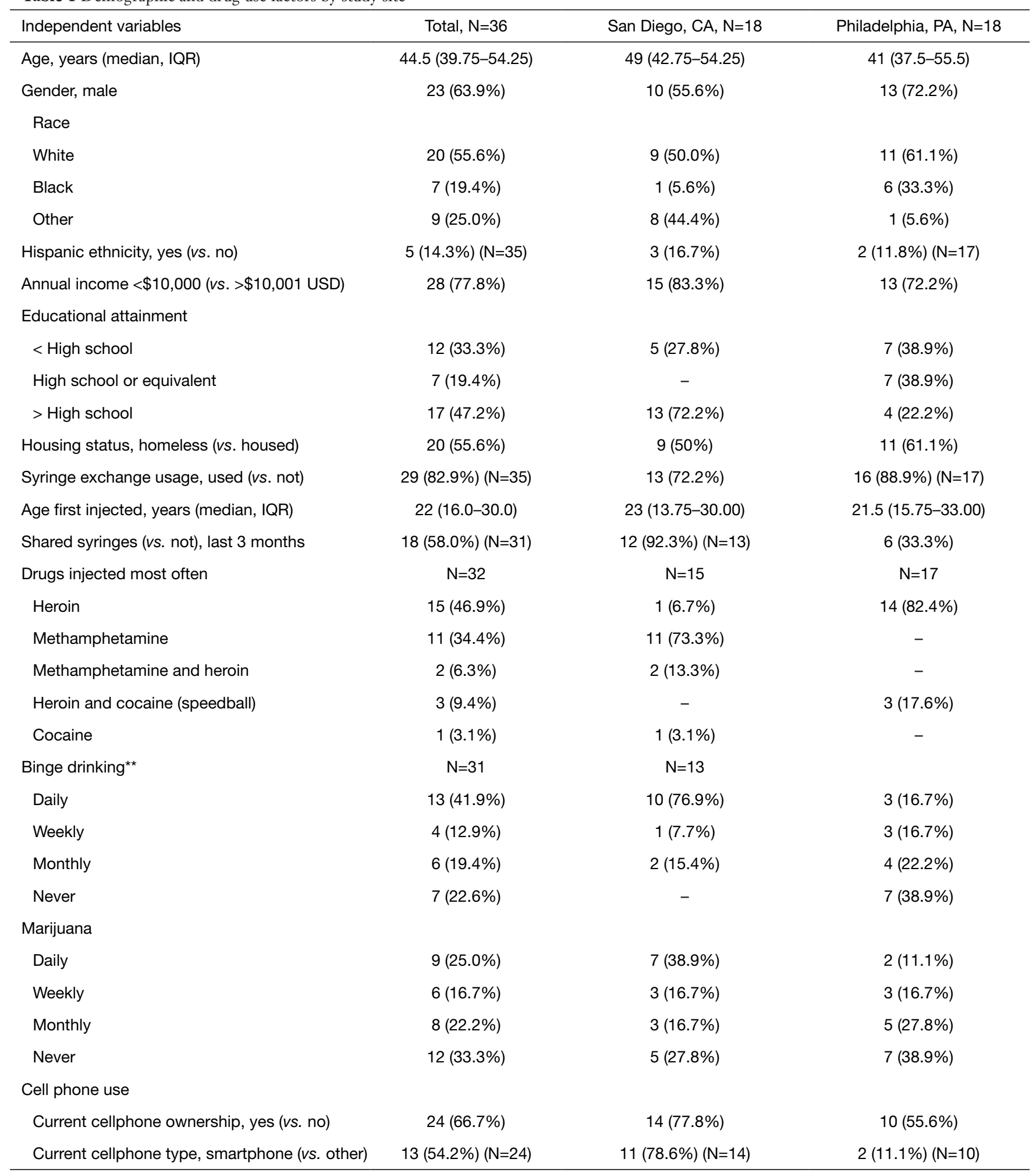

*, the recall period for questions was 6 months in San Diego and 3 months in Philadelphia; **, binge drinking was defined as consuming 5 or more drinks in a single day; ${ }^{\dagger}$, variations in $\mathrm{N}$ for demographic and drug use factors is due to missing or incomplete data for the indicated variable. 


\section{Potential catalyst for behavior change}

For many participants, increased self-reflection was considered a potential catalyst for behavior change, both by encouraging participants to alter behaviors they perceived as negative (e.g., drug use, sexual risk behaviors and poor medication adherence), and/or by reinforcing positive behaviors (e.g., medication adherence). A 39-year-old male participant described:

"If I'm better prepared or if I have more information on why I'm doing the things that I do, then I can start trying to avoid them...maybe pull myself out of the situation that I am in. I think that would be very belpful... (I'm interested in tracking my behavior) to see some of the stuff I really do want to change... keeping track of it, keeping it more refreshed in my mind instead of doing it and forgetting about it. Being forced to have to look at it (my behavior) may make me want to change some of the stuff that I've been doing."

A 58-year-old female participant described how reporting anti-retroviral medication adherence was empowering. She also described how repeatedly responding to adherence questions might be a cue to action to improve medication taking behavior for someone struggling to take their medications as prescribed:

"I feel good when I realized I can say I take (my medication) every day no matter what...It empowers me. If someone wasn't taking their meds like they're supposed to every day, I would hope that that question would make them stop and think and make them realize that they need to do a little better if they want to be around. They need to do a lot better and take them every day like they're supposed to."

Diary-keeping was considered both a reinforcement of positive behavior as well as a possible cue to action for those who may not consistently engage in health behavior such as medication adherence, practicing safer sex and drug use, and maintaining sobriety after participating in a detox program.

A 47-year-old female described how completing the simulations allowed her to report her healthy behavior, which in turn might reinforce those efforts:

"The questions make me conscious...like I guess a lot of people don't use condoms, they share their needles, they share their waters...I'm a real stickler for not sharing anything...So when I go through (the simulations) it makes me more conscious of the fact that I'm actually doing something right! Because even though I'm doing something wrong like using, at least I'm keeping myself bealthy and clean."

Similarly, a 40-year-old female stated, "If this one time (completing the simulation) impacted me and had me thinking, if I did it through the whole month, then that could be a lifechanging thing...That might help me more to where when I do go to detox, that would help me...stick in my mind and keep me clean and sober longer."

\section{Catharsis}

Many participants recognized that recording their activities and mood in an EMA diary may serve as a way to release emotions that they were previously holding inside. Not releasing emotions was perceived to be negative by participants. A 39-year-old male described why he felt comfortable talking about his drug use when he said, "Because if you keep things closed up in your head, it's not healthy for you." The ability to release emotions was seen as a benefit of participating in the study, and could potentially relieve stress. A 50-year-old female stated, "Doing the EMAs would bring me down. Alleviate a lot of stress... I just heard that writing thoughts down is a good outlet for you, for your stress."

Some participants felt that thinking and writing about their drug use could be a way of helping to deal with drug cravings. A 34-year-old female stated:

"Writing about cravings didn't make it worse. Getting it out I think it helps a lot. Fust even putting it out there like, 'I want to use.' If you want to use, just saying it out loud, saying it to someone takes a lot of the weight off you. If you're keeping it in your head on your own then you're going to use. And that's a problem. I certainly hope that if you just keep it all inside and things like that, then [doing the EMAs] is a way to get it out...It would be a good thing if anything, definitely."

\section{Altruism}

Participants frequently reported that sharing their experiences via the EMAs might in turn benefit others by giving researchers information needed to create programs and policies that benefit their community. Participants often cited altruistic feelings as both motivations to and benefits of participating in this and future research. A 65-year-old male described:

"Participating in this study may give you the chance to maybe save someone's life. Maybe there's one thing that I'll say through your survey and whatever you all taking and do with it might belp someone...You never know. It's gonna belp, I pray to God, that it helps somebody."

For many, feeling like they were helping others was a personal benefit. A 40-year-old male described, "Answering 
the EMA questions doesn't bother me...It's gonna belp others, it's gonna belp the doctors try to figure out why we drug addicts use, and try to prevent us from doing it. It kinda makes me feel good that I'm actually helping in a way."

Feeling that their knowledge and expertise has the potential to help others and to contribute to science in general may also enhance participants' sense of self-worth. A 40-year-old male described, "Doing the EMAs makes me feel important, like I'm worth something. My opinion counts." Similarly, a 39-year-old male remarked: "You get a person like me...they won't mind doing the study... Most of the time we out bere using... We're not really doing anything important. So it would make a person feel important."

\section{Social support via cell phone access}

Participants reported that the use of a cell phone for the period of the proposed EMA study would be a great benefit to their daily lives in terms of social support. Several participants noted that being provided a cell phone with service would allow them to remain in better contact with their families. A 22-year-old male reflected: "I'd call my mom and my grandma more than once a week. I used to, when I had a phone, talk to my grandma every day. We are very close... I'd call her every day. I'd talk to my mom, text my mom every day. Now I have to call them from payphones and stuff and we can only talk for like 3 minutes."

Similarly, a 55-year-old male remarked, "Having the phone would...make better relationships with my family. I would be able to get in touch with them. I would be able to talk to them."

In addition to the phone's hypothesized ability to keep participants better connected to family, having a phone with wireless internet could serve as a way of connecting participants to a world outside of their immediate surroundings, which participants viewed as beneficial. A 47-year-old female stated:

"The internet alone is something that people enjoy. That keeps them...able to share with other people around the world. Like right now, everybody is stuck in this little dome so to speak, and nobody can get out of it. This neighborbood is like a black bole, once you fall in, it's real hard to get out. People need to broaden their horizons and be able to explore things."

\section{Discussion}

Data from this study suggests that participating in an EMA study of polydrug use may provide several forms of benefits to participants. One type of benefit is therapeutic in nature stemming from repeatedly answering questions related to their sex and drug behaviors. In longitudinal EMA studies, this phenomenon is sometimes referred to as assessment reactivity. Participants hypothesized repeated behavioral assessments would encourage self-reflection, which in turn could lead to behavior change. One possible result of their reflection is the reinforcement of positive behaviors and/ or modification of behaviors perceived by participants to be negative. Their observation of the potential intervention effects from these assessments is in line with other research (27-30). Gunn and colleagues reported that participation in an exploratory 4-week EMA and interview study of sexual behavior of women engaging in transactional sex had the unanticipated effect of increasing self-esteem and lowering depression and anxiety scores (30). Similarly, Stopka et al. reported that persons who inject drugs and who completed daily diaries related to syringe activities experienced an unexpected intervention effect; several participants in the study expressed a desire to quit using drugs in their diaries and three diarists enrolled in a drug treatment program during the course of the diary study or shortly thereafter (31).

One explanation for the potential intervention effect is that EMAs may serve as cues to action. Cues to action is one of the six key concepts in the Health Belief Model, which is a framework that posits the desire to avoid a negative health consequence is a prime motivator of individuals' health behavior when the benefits of enacting the behavior outweigh the potential barriers (32). According to the model, a stimulus, or cue to action, must be present in order to trigger the health-promoting behavior. Cues to action include providing information, promoting awareness, and providing reminders and have been used in a number of studies to support engagement in healthy behaviors (33) such as adherence to anti-retroviral medications $(34,35)$, smoking cessation (36), and weight loss (37), to name just a few. Researchers using EMA should be aware of potential intervention effects of using this method and plan studies in a way that promotes teasing out intervention effects.

In part, participants reported that their ability to selfreflect would be heightened from using a cell phone to report sensitive and stigmatized behavior. Repeatedly, participants commented on the key benefits of direct data entry into the cell phone as opposed to in-person reporting. Other studies where data is collected via technology, such as those using computer assisted self-interviewing (CASI) and audio computer assisted self-viewing (ACASI), have reported participants feel more comfortable disclosing 
stigmatized behavior to a computer than an in-person interview (38-40). Decreasing social desirability bias using CASI/ACASI has been shown to improve validity in sexual and drug behavior research (41-43). Thus, the use of technology in EMA research benefits not only the participant by facilitating disclosure of honest answers that could benefit them via self-reflection, and the study as a whole through the collection of more accurate data.

In line with research related to benefits of nonEMA research, participants in our simulations expressed altruistic motivations for participation in EMA research, especially as these benefits could be conferred to their peers (44-48). Participants reflected on their potential to contribute to research that could improve the lives of other people who use drugs and to potentially save lives. Participants reported that these contributions would make them feel good and enhance their self-worth. Altruistic motivations for participation in research have been found in other studies to have a positive influence on the lives of participants through increased physical health and decreased depression, anxiety, and insomnia symptomology (49). This is hypothesized to be due to feelings of interconnectedness with others, displacement of negative emotions, and promoting a sense of purpose (49). Other authors have found that, among persons who inject drugs, altruism and the desire to help others is a key driver of research participation $(46,50)$.

Taken together, findings from our study add to the ongoing discussion about assessment reactivity within EMA research $(20,21,23)$. Participants from our study anticipated that repeated assessment of mood and behavior could change their drug-related behavior. While one limitation of our study is that we cannot test for these effects, this study uniquely contributes to the literature by offering theoretical explanations for how assessment reactivity may occur: repeated behavioral assessments could encourage selfreflection, which could lead to behavior change; EMA may serve as cues to action to practice safer sex and drug behavior; or the benefit could stem from simply participating in any research, and feeling good about contributing to research that may help their peers. We believe measurement of these effects over time through the use of an appropriate design that accounts for randomization of subjects into intervention and control groups, and long-term follow-up of participants to assess for sustained benefits over time are important next steps to move the field of EMA forward.

While participants reported potential benefits of having access to a study-provided cell phone, potential ethical concerns that may arise related to the provision of cell phones in an EMA study must be mentioned. As noted by Labrique and colleagues [2013], by using smartphone technology in a research study, researchers may be enacting selection bias by only enrolling technologically literate participants (16). This may violate the ethical principle of fair selection. Investigators should explore the acceptability of EMA research among potential participants with low technological literacy and whether/how these persons may be trained to use technology. Second, having access to a cell phone with internet access was named as a benefit by multiple participants. If these phones are to be returned to investigators or data suspended at a study's conclusion, this removal of a benefit could have a negative impact on participant lives. For example, in our study, only 13 out of 38 (36\%) of participants owned a smartphone at the time of the study, so removing the study-provided cell phone with internet access could be a potential harm to a large subset of the sample. Further research is needed to assess potential harms of removing benefits, such as the study provided cell phone, once research ends. One possible solution would be to allow participants to retain the device, once any confidential information has been removed, and allowing them to continue cell phone service at their own cost. This potentially would have been helpful for the $33.3 \%$ of the participants in this study who did not currently own a cell phone, though feasibility of paying for cell phone service needs to be explored.

Like any study, our findings must be interpreted in the light of our limitations. Our study was exploratory in nature and included a sample of 36 participants. Because the participants' feedback of completing an EMA diary entry was a simulation, benefits reported by participants are hypothetical in nature, and may not reflect what would actually occur in an EMA study. Future EMA studies should include measurements to identify if participants received benefits as a result of the assessments, and if the benefits are sustained over time. Additionally, while this study focused on the perceived potential benefits of participating in an EMA study, potential benefits need to be weighed against the perceived potential risks of EMA research, which were mostly related to legal and social concerns about data collection and security, as more thoroughly described elsewhere (18). Participants in our study were engaging in high-risk polydrug use. Evidence suggests, even when compared to other types of illicit drug users, polydrug users are a very stigmatized and marginalized group who may engage in more frequent high-risk drug use $(51,52)$. 
It is possible that they identified potential benefits that are different from what other populations may relate to, thereby limiting the generalizability of our findings. Lastly, the EMA simulation was followed by a semi-structured interview. This study cannot determine if the potential benefits reported stemmed from completing the EMA itself or from being interviewed about the experience. Research suggests therapeutic benefits from both types of data collection. Future studies could better tease out the root of this effect through the use of multiple or cross over intervention arms by asking participants to clearly delineate their experience.

Our findings suggest that EMA is perceived to be potentially beneficial to persons engaging in high-risk polydrug use. While some may argue that high intensity studies like EMA may pose risks to participants because of constant reflection of stigmatized behaviors, our participants suggest that it is this constant assessment that would lead to self-reflection. Self-reflection, in turn, has the potential to help participants engage in healthy behavior or reinforce positive behavior changes. Across multiple domains, findings suggest that participants may derive benefits from EMA research, and that further scholarship is needed to determine how significant, consistent, and direct these benefits are. Researchers using this method need to be aware of potential benefits conferred by the method.

\section{Acknowledgements}

We would like to thank Brogan Piecara \& Dr. John Rossi for providing valuable feedback on the manuscript, the respondents who provided data for this research, and the STAHR-II and Prevention Point Philadelphia staff.

Funding: This work was supported by the National Institutes of Health (1R01 DA031074 to RS Garfein, R25 DA031608 to Celia Fisher).

\section{Footnote}

Conflicts of Interest: The authors have no conflicts of interest to declare.

Ethical Statement: The study was approved by the Drexel University and University of California San Diego Institutional Review Boards (No. 3192 and No. 3113 at Drexel University, and 3192 at UCSD) and written informed consent was obtained from all patients.

\section{References}

1. Shiffman S. Ecological Momentary Assessment (EMA) in Studies of Substance Use. Psychol Assess 2009;21:486-97.

2. Shiffman S, Hickcox M, Paty JA, et al. Progression from a smoking lapse to relapse: prediction from abstinence violation effects, nicotine dependence, and lapse characteristics. J Consult Clin Psychol 1996;64:993.

3. Cooper ML, Russell M, Skinner JB, et al. Stress and alcohol use: moderating effects of gender, coping, and alcohol expectancies. J Abnorm Psychol 1992;101:139.

4. Paty J, Kassel J, Shiffman S. Assessing stimulus control of smoking: The importance of base rates. In: DeVries MW. editor. The experience of psychopathology: investigating mental disorders in their natural setting. New York: Cambridge University Press, 1992:347-52.

5. Shiffman S, Stone AA, Hufford MR. Ecological Momentary Assessment. Annu Rev Clin Psychol 2008;4:1-32.

6. Roth AM, Hensel DJ, Fortenberry JD, et al. Feasibility and acceptability of cell phone diaries to measure HIV risk behavior among female sex workers. AIDS Behav 2014;18:2314-24.

7. Comulada WS, Swendeman D, Wu N. Cell phone-based ecological momentary assessment of substance use context for Latino youth in outpatient treatment: Who, what, when and where. Drug Alcohol Depend 2016;167:207-13.

8. Comulada WS, Lightfoot $M$, Swendeman D, et al. Compliance to cell phone-based EMA among Latino youth in outpatient treatment. J Ethn Subst Abuse 2015;14:232-50.

9. Benarous X, Edel Y, Consoli A, et al. Ecological momentary assessment and smartphone application intervention in adolescents with substance use and comorbid severe psychiatric disorders: study protocol. Front Psychiatry 2016;7:157.

10. Serre F, Fatseas M, Swendsen J, et al. How psychiatric comorbidity and mood states influence craving and substance use in daily life? An ecological momentary assessment study in patients with alcohol, tobacco, cannabis and heroin use disorders. Drug \& Alcohol Dependence 2017;171:e187.

11. Epstein DH, Willner-Reid J, Vahabzadeh M, et al. Realtime electronic diary reports of cue exposure and mood in the hours before cocaine and heroin craving and use. Arch Gen Psychiatry 2009;66:88-94.

12. Freedman MJ, Lester KM, McNamara C, et al. Cell phones for ecological momentary assessment with cocaineaddicted homeless patients in treatment. J Subst Abuse 
Treat 2006;30:105-11.

13. Hopper JW, Su Z, Looby AR, et al. Incidence and patterns of polydrug use and craving for ecstasy in regular ecstasy users: An ecological momentary assessment study. Drug Alcohol Depend 2006;85:221-35.

14. Johnson EI, Barrault M, Nadeau L, et al. Feasibility and validity of computerized ambulatory monitoring in drugdependent women. Drug Alcohol Depend 2009;99:322-6.

15. Capon H, Hall W, Fry C, et al. Realising the technological promise of smartphones in addiction research and treatment: an ethical review. International Journal of Drug Policy 2016;36:47-57.

16. Labrique AB, Kirk GD, Westergaard RP, et al. Ethical issues in mHealth research involving persons living with HIV/AIDS and substance abuse. AIDS Res Treat 2013;2013:189645.

17. Committee on Revisions to the Common Rule for the Protection of Human Subjects in Research in the Behavioral and Social Sciences; Board on Behavioral, Cognitive, and Sensory Sciences; Committee on National Statistics; Committee on Population; Division of Behavioral and Social Sciences and Education; National Research Council. Proposed revisions to the common rule for the protection of human subjects in the behavioral and social sciences. Washington (DC): National Academies Press (US), 2014.

18. Roth AM, Rossi J, Goldshear JL, et al. Potential Risks of Ecological Momentary Assessment Among Persons Who Inject Drugs. Subst Use Misuse 2017;52:840-7.

19. Lyubomirsky S, Sousa L, Dickerhoof R. The costs and benefits of writing, talking, and thinking about life's triumphs and defeats. J Pers Soc Psychol 2006;90:692-708.

20. Epstein EE, Drapkin ML, Yusko DA, et al. Is alcohol assessment therapeutic? Pretreatment change in drinking among alcohol-dependent women. J Stud Alcohol 2005;66:369-78.

21. Clifford PR, Maisto SA, Davis CM. Alcohol treatment research assessment exposure subject reactivity effects: part I. Alcohol use and related consequences. J Stud Alcohol Drugs 2007;68:519-28.

22. Hufford MR, Shields AL, Shiffman S, et al. Reactivity to ecological momentary assessment: An example using undergraduate problem drinkers. Psychol Addict Behav 2002;16:205-11.

23. McCarthy DE, Minami H, Yeh VM, et al. An experimental investigation of reactivity to ecological momentary assessment frequency among adults trying to quit smoking. Addiction 2015;110:1549-60.
24. Robertson AM, Garfein RS, Wagner KD, et al. Evaluating the impact of Mexico's drug policy reforms on people who inject drugs in Tijuana, BC, Mexico, and San Diego, CA, United States: a binational mixed methods research agenda. Harm Reduct J 2014;11:4.

25. Drennan J. Cognitive interviewing: verbal data in the design and pretesting of questionnaires. J Adv Nurs 2003;42:57-63.

26. Zhao T, McDonald S, Edwards HM. The impact of two different think-aloud instructions in a usability test: a case of just following orders? Behav Inf Technol 2014;33:163-83.

27. Campbell R, Sefl T, Wasco SM, et al. Doing community research without a community: creating safe space for rape survivors. Am J Community Psychol 2004;33:253-61.

28. Kurtz SP, Surratt HL, Buttram ME, et al. Interview as intervention: the case of young adult multidrug users in the club scene. J Subst Abuse Treat 2013;44:301-8.

29. Pennebaker JW, Graybeal A. Patterns of Natural Language Use: Disclosure, Personality, and Social Integration. Cur Dir Psy 2001;10:90-3.

30. Gunn JK, Roth AM, Center KE, et al. The Unanticipated Benefits of Behavioral Assessments and Interviews on Anxiety, Self-Esteem and Depression Among Women Engaging in Transactional Sex. Community Ment Health J 2016;52:1064-9.

31. Stopka TJ, Springer KW, Khoshnood K, et al. Writing About Risk: Use of Daily Diaries in Understanding DrugUser Risk Behaviors. AIDS Behav 2004;8:73-85.

32. Rosenstock IM. Historical origins of the health belief model. Health Educ Monogr 1974;2:328-35.

33. Van Der Drift EJ, Beun RJ, Looije R, et al. A remote social robot to motivate and support diabetic children in keeping a diary. Proceedings of the 2014 ACM/IEEE international conference on Human-robot interaction. 2014: ACM.

34. Swendeman D, Ramanathan N, Baetscher L, et al. Smartphone self-monitoring to support self-management among people living with HIV: perceived benefits and theory of change from a mixed-methods randomized pilot study. J Acquir Immune Defic Syndr 2015;69 Suppl 1:S80-91.

35. van Velthoven MH, Brusamento S, Majeed A, et al. Scope and effectiveness of mobile phone messaging for HIV/ AIDS care: a systematic review. Psychol Health Med 2013;18:182-202.

36. Whittaker R, Borland R, Bullen C, et al. Mobile phonebased interventions for smoking cessation. Sao Paulo Medical Journal 2010;128:106-7.

37. Wei J, Hollin I, Kachnowski S. A review of the use of mobile phone text messaging in clinical and healthy 
behaviour interventions. J Telemed Telecare 2011;17:41-8.

38. Estes LJ, Lloyd LE, Teti M, et al. Perceptions of audio computer-assisted self-interviewing (ACASI) among women in an $\mathrm{HIV}$-positive prevention program. PLoS One 2010;5:e9149.

39. Dolezal C, Marhefka SL, Santamaria EK, et al. A Comparison of Audio Computer-Assisted Self-Interviews to Face-to-Face Interviews of Sexual Behavior Among Perinatally HIV-Exposed Youth. Arch Sex Behav 2012;41:401-10.

40. Oschwald M, Leotti S, Raymaker D, et al. Development of an audio-computer assisted self-interview to investigate violence and health in the lives of adults with developmental disabilities. Disabil Health J 2014;7:292-301.

41. Hewett PC, Mensch B, Erulkar A. Consistency in the reporting of sexual behaviour by adolescent girls in Kenya: a comparison of interviewing methods. Sex Transm Infect 2004;80:ii43-8.

42. Kissinger P, Rice J, Farley T, et al. Application of computer-assisted interviews to sexual behavior research. Am J Epidemiol 1999;149:950-4.

43. Turner CF, Ku L, Rogers SM, et al. Adolescent sexual behavior, drug use, and violence: increased reporting with computer survey technology. Science 1998;280:867-73.

44. Hutchinson SA, Wilson ME, Wilson HS. Benefits of Participating in Research Interviews. Image J Nurs Sch

doi: 10.21037/mhealth.2017.10.01

Cite this article as: Roth AM, Felsher M, Reed M, Goldshear JL, Truong Q, Garfein RS, Simmons J. Potential benefits of using ecological momentary assessment to study high-risk polydrug use. mHealth 2017;3:46.
1994;26:161-4.

45. Felsher M, Wiehe SE, Gunn JK, et al. 'I Got it off my Chest': An Examination of how Research Participation Improved the Mental Health of Women Engaging in Transactional Sex. Community Ment Health J 2017. [Epub ahead of print].

46. Fry C, Dwyer R. For love or money? An exploratory study of why injecting drug users participate in research. Addiction 2001;96:1319-25.

47. McDonald KE, Kidney CA, Patka M. 'You need to let your voice be heard': research participants' views on research. J Intellect Disabil Res 2013;57:216-25.

48. Beck CT. Benefits of Participating in Internet Interviews: Women Helping Women. Qual Health Res 2005;15:411-22.

49. Post SG. Altruism, happiness, and health: It's good to be good. Int J Behav Med 2005;12:66-77.

50. Friedman SR, Pouget ER, Sandoval M, et al. Measuring altruistic and solidaristic orientations toward others among people Who inject drugs. J Addict Dis 2015;34:248-54.

51. Kokkevi A, Kanavou E, Richardson C, et al. Polydrug use by European adolescents in the context of other problem behaviours. Nordic Studies on Alcohol and Drugs 2014;31:323-42.

52. Rafful C, Wagner KD, Werb D, et al. Prevalence and correlates of neck injection among people who inject drugs in Tijuana, Mexico. Drug Alcohol Rev 2015;34:630-6. 